\title{
Brucelosis: zoonosis de importancia en México
}

\author{
Rosa Lilia Guzmán-Hernández, Araceli Contreras-Rodríguez, \\ Eric Daniel Ávila-Calderón y M. Rosario Morales-García
}

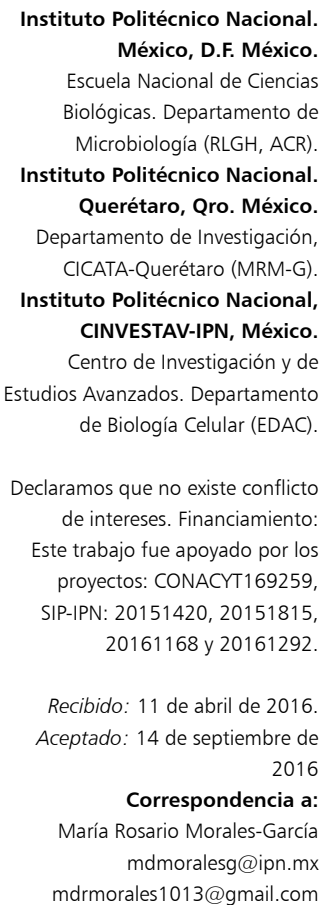

\section{Introducción}

L a brucelosis es una zoonosis mundial con impacto, tanto en la salud pública en humanos como en la salud animal, que genera pérdidas económicas en la industria ganadera. Esta zoonosis ha sido controlada en los países con ingresos altos, no así en los países en desarrollo. Se señala que cada año se presentan 500.000 nuevos casos de brucelosis humana en todo el mundo. México ocupó en el año 2006 el vigésimo primer lugar mundial en brucelosis humana y el segundo lugar en el continente americano, con una incidencia de 1,74 casos por cada 100.000 habitantes, la que aumentó a 2,97 para el año $2011^{1,2}$. En los últimos cinco años esta enfermedad se ha asociado con las condiciones socio-económicas desfavorables de la población ${ }^{3,4}$.

\section{David Bruce y Brucella}

Conocida antiguamente como fiebre de Malta, fiebre del Mediterráneo, fiebre ondulante, enfermedad de Bang, y más recientemente nombrada por el organismo que la produce, la brucelosis es considerada una de las zoonosis más relevantes en el concierto mundial hoy en día. A David Bruce se le atribuye la identificación de Brucella durante su estancia en la isla de Malta, donde fue enviado como cirujano del cuerpo médico de la Armada Real Británica. Malta, es una isla pequeña localizada al centro del Mediterráneo al sur de Italia, al oriente de Túnez y al norte de Libia. Bruce observó casos de una enfermedad que causaba fiebre que alcanzaba los $41^{\circ} \mathrm{C}$ en las noches y que se normalizaba en el día. En el hospital de Valetta donde se instaló, no contaba Bruce con laboratorios de investigación; no obstante, alentado por el trabajo que Robert Koch había realizado sobre el descubrimiento del bacilo de la tuberculosis, decidió investigar el agente causal de la fiebre de Malta, enfermedad bastante común entre los soldados de la tropa británica ${ }^{5}$. Comenzó por adquirir un microscopio, donde observó cocos muy pequeños en el bazo de un soldado que murió a causa de la enfermedad. Posteriormente logró aislar el "micrococo" siguiendo los postulados de Koch y reportó sus observaciones en 1887. Él pudo reproducir la enfermedad en monos, quienes mostraron fiebre y algunos de ellos murieron. Bruce nombró a la bacteria con el nombre de Micrococcus melitensis $^{6}$. En 1896, Bang aisló a la bacteria que generaba aborto en vacas y la nombró Bacillus abortus. Posteriormente en 1914 Traum, aisló una bacteria de un aborto de cerdo que posteriormente se nombró como $B$. suis. En 1918, Alice Evans, estableció una conexión entre Micrococcus melitensis y Bacillus abortus. Así mismo, la Dra. Evans indicó que esta enfermedad de los animales de crianza, no ocasionaría contagio alguno al humano, si se consumiera su leche pasteurizada ${ }^{7}$. Feusier y Meyers en 1920, nombraron al género Brucella en honor a Sir David Bruce, microbiólogo y patólogo escocés que identificó al microorganismo por primera vez. La epidemiología de la fiebre de Malta era desconocida hasta 1905 cuando Temistocles Zammit, uno de los miembros malteses de la Comisión para la Investigación de la Fiebre del Mediterráneo encabezada por el Dr. Bruce, encontró que la 
leche de cabra era el vehículo que diseminaba la bacteria. Cuando la leche de cabra se eliminó de la dieta de las tropas británicas en Malta, la enfermedad se controló en los soldados ${ }^{5}$. Hoy en día, a más de 100 años de que Zammit asociara a Brucella con la leche de cabra, la fiebre de Malta sigue causando enfermedad tanto en animales como en el hombre en varias regiones del planeta. Sin embargo, países como Canadá, E.U.A., Japón, el norte y centro de Europa, Australia y Nueva Zelandia han logrado erradicar esta enfermedad ${ }^{8,9}$.

\section{Las especies del género Brucella}

Se conoce que las especies de Brucella tienen predilección para establecerse en ciertos hospederos, por ejemplo: B. melitensis infecta a caprinos, B. abortus infecta ganado vacuno, y B. suis infecta cerdos. Sin embargo, puede darse la infección cruzada, es decir, $B$. melitensis puede infectar vacas o cerdos, o B. abortus podría infectar cabras ${ }^{10}$. Además de estas tres especies, se identificaron otras más, como: $B$. canis que infecta perros, $B$. ovis que se asocia con la infección a ovinos, y $B$. neotomae que se aisló de una rata del desierto. Éstas conforman las seis especies clásicas que componen al género Brucella. Sin embargo, en las últimas décadas se han identificado otras especies, entre ellas: $B$. microti que se aisló de roedores endémicos de la República Checa en el año 2001, siendo reconocida en 2008 como nueva especie $^{11}$. En 1994, se describió el aislamiento de Brucella sp. en focas, marsopas, y delfines en Escocia ${ }^{12}$. Después del análisis genético de diferentes aislados procedentes de mamíferos marinos, se propusieron dos nuevas especies: $B$. pinnipedialis y $B$. ceti ${ }^{13}$. Posteriormente se identificó una nueva especie, denominada $B$. inopinata, la que fue aislada de un implante mamario ${ }^{14}$. En 2014 se aisló Brucella, de mandriles, a la que se ha propuesto nombrarla como B. papionis ${ }^{15}$. Recientemente, a partir de muestras de nódulos linfáticos de zorros rojos en Austria, se aisló una nueva especie la que fue descrita como: $B$. vulpis ${ }^{16}$. Por otra parte, se han reportado anticuerpos contra Brucella en animales silvestres como alces, búfalos, venados y cerdos salvajes $^{12}$. Por lo que estos animales silvestres se consideran como reservorios de la bacteria.

\section{Características de la bacteria}

Brucella es un bacilo gramnegativo, que carece de los factores de virulencia clásicos que se han reportado en otros gramnegativos, tales como: toxinas, flagelos, etc. A pesar de esto, es una bacteria asombrosamente virulenta. En animales de experimentación se ha reportado que su dosis mínima infectante, va desde 10 hasta 100 células, ya sea por vía aerosoles o por vía subcsutánea ${ }^{17}$.
Debido a su capacidad de formar fácilmente aerosoles, se encuentra en la lista de bacterias, que pueden ser utilizadas en bioterrorismo, y debido a ello, en algunos países está restringido trabajar con Brucella, tanto en laboratorios clínicos, de investigación o en los que se realiza la producción de vacunas para animales ${ }^{18,19}$. De acuerdo con los Centros para el Control y Prevención de Enfermedades (CDC) de Atlanta, E.U.A., Brucella debe manipularse en laboratorios de bioseguridad nivel 3 y el personal que ahí labore deberá ser altamente capacitado ${ }^{20}$. Varios reportes en la literatura científica consideran a Brucella como un organismo de alto riesgo, ya que al ser manipulada en el laboratorio, sumado generalmente a errores de su manipulación, contamina el personal ${ }^{21}$. El principal mecanismo de infección en el laboratorio, es la quema del asa, que al contener al microorganismo, puede generar aerosoles, y así ingresa por la conjuntiva. Otro error de manipulación, es el pipetear fluidos contaminados con la boca, o la manipulación de las muestras sin guantes, ya que la bacteria puede ingresar al cuerpo humano por la boca o a través de heridas en la piel.

Es importante mencionar que en la actualidad no se cuenta con vacunas para humanos ${ }^{22}$.

\section{Brucella evade al sistema inmune del hospedero}

Otra de las características importantes de la bacteria, es su capacidad de residir en el espacio intracelular, por lo que se le considera como una bacteria intracelular facultativa. Esto significa que es capaz de residir dentro de células fagocíticas y no fagocíticas, aunque también puede sobrevivir fuera de las células, y aislarse de la leche cruda o sus derivados. Los macrófagos ${ }^{23}$ los internalizan dentro de su citoplasma, rodeándoles por un fragmento de la membrana, denominado fagosoma. Una vez dentro, el fagosoma se fusiona con los lisosomas, los que vierten al interior de esta ultraestructura enzimas que tienen como función destruir a la bacteria. El fagosoma también posee un $\mathrm{pH}$ ácido que le ayuda a eliminar a las bacterias $^{24}$. Brucella es capaz de evitar la fusión del fagosoma con los lisosomas, además de soportar el $\mathrm{pH}$ ácido del fagosoma e incluso, el $\mathrm{pH}$ ácido las estimula a producir y secretar proteínas que le permiten sobrevivir y evadir los diversos mecanismos antimicrobianos que poseen los macrófagos ${ }^{25}$. Durante las primeras 48 h, 90\% de las brucelas que infectan a un humano son eliminadas, pero aquellas que evitan los mecanismos bactericidas de los macrófagos y quedan contenidas en éstos, modifican la señalización intracelular de estas células eucariotas, con lo que logran dirigirse hacia el retículo endoplásmico donde residirán en una vacuola llamada: vacuola contenedora de Brucella (BCV por sus siglas en inglés). Constituyen de 
esta forma, el nicho intracelular para su supervivencia a largo plazo, en el que inician su replicación y propagación por todo el organismo, viajando así de célula a célula ${ }^{26}$. Brucella tiene mecanismos evolutivos sofisticados para evadir al sistema inmune de los mamíferos que infecta, por lo que, puede causar una enfermedad en un estado latente asintomático con reactivación tardía del sistema inmunológico ${ }^{27}$. Durante la etapa aguda, generalmente se desencadenan cuadros febriles ocasionados esencialmente por la respuesta de tipo inflamatoria (producción de citoquinas pro-inflamatorias). A las seis semanas de infección se pueden detectar anticuerpos contra Brucella spp., siendo del tipo IgM, $\operatorname{IgG}_{1}, \operatorname{IgG}_{2}$ e $\operatorname{IgG}_{3}$. La infección no genera una memoria inmunológica, debido a que la respuesta inmunológica humoral en contra de infecciones intracelulares es limitada y no protectora ${ }^{26}$. Y aunque los anticuerpos juegan un papel en la resistencia a la infección, el principal mecanismo de eliminación de la bacteria es a través de una respuesta inmunológica celular ${ }^{28}$.

\section{El impacto de la brucelosis en la ganadería}

Los animales enfermos son la principal fuente de dispersión de la bacteria; un animal enfermo en un rebaño puede fácilmente diseminar la enfermedad en los animales sanos o incluso en rebaños cercanos. La infección cruzada puede también generar otra problemática, debido a que se ha reportado que el ganado vacuno puede infectarse con $B$. melitensis; en ese caso, el manejo del ganado requiere un cuidado especial ${ }^{29}$. La infección con Brucella en los machos genera inflamación de los testículos y la bacteria se almacena en sus vesículas seminales, el ámpula y el epidídimo. $\mathrm{Y}$ en el caso de las hembras preñadas, les provoca aborto debido a que la bacteria se localiza en la placenta, donde se produce una placentitis grave con infección del feto, ocasionando así el aborto. En el caso de las vacas lecheras la producción de la leche se ve minimizada; además se ha demostrado que un gran porcentaje de vacas infectadas libera a la bacteria por la leche ${ }^{10}$. Todo esto conlleva a grandes pérdidas económicas en la industria ganadera, principalmente por los abortos, la retención placentaria, la disminución de la producción lechera y el alumbramiento de becerros con debilidad y bajo peso al nacer ${ }^{30}$. La problemática se agudiza porque un animal que se diagnostica con brucelosis, es un animal que debe ser sacrificado según lo establecido por recomendaciones internacionales ${ }^{31}$. El sacrificio de los animales infectados en los países en vías de desarrollo, es una medida muy difícil de llevar cabo, debido a que particularmente los pequeños productores, que dependen económicamente de la producción lechera o de quesos, se oponen al sacrificio. Para poder cumplir con el sacrificio se requiere que el gobierno apoye económicamente a los ganaderos, y además se implemente la vacunación masiva del ganado ${ }^{32,33}$.

Brucella melitensis (biovars 1, 2 o 3 ) es la especie que principalmente afecta a cabras y borregos en la mayor parte del mundo ${ }^{34}$. También es la especie que genera los cuadros clínicos más graves en humanos. El cerdo es un animal que también puede trasmitir brucelosis al humano, aunque existen pocos reportes de humanos infectados por B. suis.

\section{¿De qué manera se puede infectar el hombre con Brucella?}

El hombre adquiere la bacteria, a través del contacto con animales infectados; se ha reportado que en la actividad de rastros o frigoríficos, los trabajadores que presentan heridas en sus manos corren el riesgo de infectarse de brucelosis, al manipular la carne o vísceras de animales contaminados por la bacteria. En el caso de los trabajadores de ranchos, que limpian los establos de animales infectados con brucelosis, corren el riesgo de infectarse vía conjuntival, debido a que al remover los desechos de los animales, se generan aerosoles que contienen al microorganismo ${ }^{35}$. Los médicos veterinarios son también, un grupo de alto riesgo de contagio, ya que su trabajo incluye la revisión, atención y vacunación a los animales con brucelosis ${ }^{36}$. Como se mencionó anteriormente, otro sector con alto riesgo de contagio es el de las personas que manipulan muestras de fluidos biológicos, tanto en laboratorios de diagnóstico clínico como de investigación o de elaboración de vacunas ${ }^{18}$. Sin embargo, la vía más común por la que se puede adquirir la bacteria en población abierta, es por el consumo de derivados lácteos no pasteurizados: leche cruda y quesos frescos sin pasteurizar, sobre todo de origen caprino y bovino. Los derivados lácteos no pasteurizados son también un factor de riesgo para viajeros que han visitado países endémicos como España, Italia, Egipto, México o Grecia, en los cuales se han reportado casos de brucelosis ${ }^{8,37,38}$. También se ha documentado que inmigrantes de países en desarrollo que se encuentran infectados con la bacteria, son diagnosticados con brucelosis cuando llegan a Europa o a los E.U.A. ${ }^{39,40}$.

\section{Principales rasgos clínicos y diagnóstico de brucelosis en humanos}

La brucelosis en los humanos se presenta con diferentes manifestaciones clínicas inespecíficas, incluyendo cuadros asintomáticos. Usualmente se presenta como un cuadro febril agudo, afectando a cualquier grupo de edad. Puede confundirse con otras infecciones febriles y recibir tratamiento inadecuado, por lo que la enfer- 
medad podría persistir y manifestarse como una recaída o progresar a una infección crónica localizada. Esta enfermedad compromete a cualquier órgano o tejido del cuerpo, y genera una serie de complicaciones; entre las más comunes están las osteo-articulares, hepato-biliares, de vías respiratorias, genito-urinarias, cardiovasculares, neurológicas, cutáneas, y oftálmicas ${ }^{28}$. Aun cuando se realice el diagnóstico temprano y se prescriba correctamente la terapia, alrededor de 10 a $30 \%$ de los pacientes desarrollará brucelosis crónica ${ }^{41}$.

El diagnóstico de esta enfermedad se debe basar en la historia clínica del paciente, sobre todo sí existió contacto con animales enfermos, sí visitó una zona endémica e ingirió derivados lácteos no pasteurizados ${ }^{42}$. El diagnóstico deberá incluir cultivo de sangre y las pruebas serológicas. Una de las desventajas del aislamiento, se debe a que Brucella es un microorganismo de lento crecimiento en el primo-aislamiento, por lo que la probabilidad de recuperarla desde un paciente es baja (alrededor de $30 \%$ ). Debido a este inconveniente, el diagnóstico de esta enfermedad se establece a través de los test serológicos. Las pruebas serológicas usadas en México son: como prueba de tamizaje, la aglutinación del suero con antígeno Rosa de Bengala, y como pruebas confirmatorias el SAT (por sus siglas en inglés, de serum agglutination test) y el 2-mercapto etanol (2-ME). El antígeno que se usa es una suspensión de un cultivo de B. abortus cepa 1119 , inactivada con fenol. En la prueba de SAT se hacen diluciones seriadas del suero y se adiciona el antígeno. Una prueba positiva da una aglutinación que se observa con la presencia de una malla de aglutinación en el fondo del tubo (o en los pozos de una microplaca, cuando se usa el micrométodo) y el sobrenadante es transparente. En esta prueba se detectan globalmente los anticuerpos IgM, IgG e IgA aglutinantes. En presencia de agentes fuertemente reductores, es posible detectar únicamente a los anticuerpos IgG. Tanto el 2-ME como el di-tio-treitol (DTT) son capaces de romper los enlaces disulfuro y despolimerizar al pentámero de la IgM, sin afectar la actividad aglutinante de la IgG. Esta característica del 2-ME se ha usado para detectar y cuantificar los anticuerpos IgG; el ensayo sigue la misma metodología de la prueba de SAT, sólo que en la solución de ensayo se adiciona el 2-ME. Las pruebas SAT y su complemento con 2-ME son útiles en el seguimiento clínico de los pacientes con brucelosis aguda. Sin embargo, no son útiles en períodos iniciales de la enfermedad o en casos de brucelosis crónica ${ }^{43}$.

\section{La tradición del consumo de queso fresco en México}

Al igual que en otros países, el consumo de queso fresco o leche cruda es una práctica común en varias regiones de México ${ }^{44,45}$. El consumo de estos productos es histórico, pues la leche es una fuente de proteínas utilizada por la humanidad desde tiempos remotos ${ }^{7}$. Los derivados lácteos se producen generalmente en las zonas rurales; sin embargo, también son comercializados en diferentes zonas urbanas, por lo que los casos de brucelosis no sólo se restringen a las zonas rurales. Por otra parte, existe una alerta sanitaria que informa a los turistas, que tienen como destino visitar México, el evitar el consumo de queso fresco $^{46}$. El Ministerio de Salud Mexicano estableció, a través de programas nacionales, la implementación de acciones de control sanitario y prevención de la ingesta de derivados lácteos sin pasteurizar ${ }^{47}$. No obstante, continúan reportándose anualmente en promedio 2.200 nuevos casos de brucelosis en humanos, en diferentes regiones del país $^{48}$. La información oficial de los casos de brucelosis se basa en el diagnóstico serológico por lo que no existen datos sobre las especie de Brucella asociadas a los casos humanos, o sobre cómo se infectaron los pacientes. De los pocos estudios sobre casos de brucelosis en México se reportó que el consumo de quesos frescos de origen caprino contaminados con B. melitensis biovar 1 fue el vehículo que ocasionó un brote de brucelosis en humanos en una de las comunidades rurales en la provincia de Guanajuato $^{49}$. Por otra parte, en el año 2014 se reportó que en las zonas rurales se presentaron condiciones de alto riesgo en la propagación de la enfermedad en humanos, debido a que los pequeños productores de quesos frescos no tenían recursos económicos para vacunar a sus animales o para hervir la leche con la que elaboran los quesos frescos ${ }^{50}$.

\section{Escenario mexicano de la brucelosis en humanos}

A pesar de que el gobierno mexicano implementó desde 1995, la campaña para el control y erradicación de la brucelosis en los animales aplicada en México, en el período de 2000 al 2011, se registró en todo el país un aumento en la incidencia de la brucelosis bovina pasando de 1 a $15 \%$; y en la del ganado caprino el aumento fue de 2 a $7 \%{ }^{2}$. Méndez-Lozano reportó que en este mismo período, el incremento de la brucelosis bovina se relacionaba con $15 \%$ de aumento en la incidencia de los nuevos casos de brucelosis humana. Y que, la brucelosis en caprinos, se relacionaba con $33 \%$ de aumento en los casos de humanos. Las cifras de la incidencia de brucelosis humana del Ministerio de Salud Mexicano son imprecisas, no sólo por la exclusión de casos erróneamente diagnosticados, que en la mayoría de las veces no se comprueban con las pruebas serológicas de laboratorio o con el aislamiento de la bacteria para validar el diagnóstico clínico, sino también por aquellos casos bien diagnosticados pero que no tienen seguimiento serológico y un tratamiento adecuado que garantice la eliminación de este microorganismo intracelular de los pacientes. Además, debemos de considerar que en 
esta enfermedad es común que se presenten recaídas o evolucione a un estado de cronicidad.

\section{Tratamiento antimicrobiano}

Las terapias para el tratamiento de brucelosis en humanos en México se describen en la "Guía para el diagnóstico y tratamiento del paciente con brucelosis" de la NOM 022-2012 ${ }^{43}$. En las que se indican qué terapia se debe de aplicar considerando la edad y el estado clínico del paciente. Para el primer tratamiento se recomiendan tres esquemas:

- Cotrimoxazol (320/1.600 mg/día (5 mg/kg/día-25 mg/ $\mathrm{kg} /$ día de trimetoprim y sulfa, respectivamente) por vía oral (V.O) y rifampicina $600-900 \mathrm{mg} /$ día $(20 \mathrm{mg} / \mathrm{kg}$ / día) V.O.

- Doxiciclina 100-200 mg/día (3 mg/kg/día) V.O y rifampicina 600-900 mg/día (20 mg/kg/día) V.O.

- Estreptomicina $1.000 \mathrm{mg} /$ día (14 mg/kg/día) por vía intramuscular (I.M) y tetraciclina $2.000 \mathrm{mg} /$ día (25 $\mathrm{mg} / \mathrm{kg} /$ día) V.O.

En el caso del sector de salud público, en especial en las zonas rurales de México, el único tratamiento que se aplica, para cualquier edad y estado clínico del paciente con brucelosis es: cotrimoxazol (320/1.600 mg/día (5 mg/ $\mathrm{kg} /$ día- $25 \mathrm{mg} / \mathrm{kg} /$ día, de trimetoprim y sulfa, respectivamente) V.O. y rifampicina $600-900 \mathrm{mg} /$ día $(20 \mathrm{mg} / \mathrm{kg} /$ día) V.O., por sólo 15 días. Con este esquema no se logra eliminar este patógeno intracelular, pero sí se establece un gran deterioro en la calidad de vida, de los pacientes con esta enfermedad ${ }^{51}$. En los registros oficiales, no se distinguen los casos por reinfección o recaídas de los casos nuevos, por lo que hasta este momento se desconoce el costo real de atención médica para aquellos pacientes que presentan complicaciones debidas a la brucelosis.

\section{Discusión y conclusiones}

Las medidas gubernamentales establecidas para el control microbiológico de la producción de los productos lácteos sin pasteurizar, no han sido eficientes para evitar el contagio de esta enfermedad por su consumo. A pesar de ser una enfermedad de notificación obligatoria, son muy escasos los reportes encaminados a establecer la especie de Brucella que infecta a enfermos de brucelosis, así como el determinar el impacto económico de las complicaciones que esta enfermedad, mal atendida en la población, puede originar. Al carecer de cepas aisladas de los brotes, es imposible relacionar los factores de riesgo en estudios epidemiológicos o aplicar las nuevas tecnologías de epidemiología molecular que ya se usan en otros países o, igualmente importante, realizar pruebas de susceptibilidad a antimicrobianos de manera periódica. Los esfuerzos del Ministerio de Salud Animal Mexicano (SAGARPA) en implementar campañas de control y erradicación de brucelosis en los animales en todo el país, no han logrado evitar el contagio de esta enfermedad a la población mexicana. Por lo que es importante hacer conciencia en los productores de derivados lácteos no pasteurizados, el que eviten expender estos alimentos sin pasteurizar; en la población, el consumir sólo lácteos pasteurizados, así como en las autoridades de vigilancia epidemiológica en mantener medidas estrictas para controlar la comercialización de quesos frescos no pasteurizados.

El control y erradicación de la brucelosis en México requiere de la participación de varios actores: en primer lugar, el sector encargado de la sanidad animal, quien al controlar y erradicar esta enfermedad de los animales, otorgaría mayores beneficios económicos en la comercialización de los productos lácteos; con ello los pequeños productores estarían obligados a elaborar productos pasteurizados. Y, no menos importante, la participación del sector de salud pública, tanto en la divulgación a la población sobre cómo evitar el contagio, y sobre la sintomatología más común que pueden llegar a presentar. De tal forma que se logre un diagnóstico preciso, un seguimiento clínico y un tratamiento específico en dosis y tiempo para los pacientes.

Agradecimientos. RLGH recibió beca PIFI y CONACYT. EDAC recibió beca de CONACYT y SNI-CONACYT. ACR recibió beca de COFAA-IPN, EDI-IPN y SNI-CONACYT. MRMG recibió beca de COTEPABE/PL/270/1O-IPN, COTEBAL/PL/23/12-IPN, COTEBAL/PL/41/12-IPN y EDI-IPN.

\section{Resumen}

Introducción: La brucelosis es una de las enfermedades zoonóticas más frecuentes en la mayor parte del mundo. Mientras que en los países desarrollados han logrado con éxito su control, en los países en vías de desarrollo continúa siendo un gran problema de salud pública. México continúa presentando alta incidencia anual de brucelosis en humanos, por lo que es considerado un país endémico de brucelosis. Objetivo: Describir la relación de esta zoonosis con la situación epidemiológica actual en la población de México. Material y Métodos: Consulta de reportes de investigación, estudios epidemiológicos y revisiones veterinarias, realizadas en México, a través de bases de datos como PubMed, Thompson Reuters y Meshresearch. Conclusión: El riesgo del contagio de Brucella spp. en México está asociado al consumo de productos lácteos sin pasteurizar, principalmente quesos frescos. 


\section{Referencias bibliográficas}

1.- Pappas G, Papadimitriou P, Akritidis N, Christou L, Tsianos E V. The new global map of human brucellosis. Lancet Infect Dis 2006; 6 (2): 91-9.

2.- Méndez-Lozano M, Rodríguez-Reyes E J, Sánchez-Zamorano L M. Brucellosis, a zoonotic disease present in the population: A time series study in Mexico. Salud Publica Mex 2015; 57 (6): 519-27.

3.- Oceguera M D, Frakena K, Udo H, Keilbach B N M, van der Zijpp A. Prevalence and risk factors for brucellosis in goat areas of Mexico with and without brucellosis control campaign. Trop Anim Health Prod 2013; 45: 1383-9.

4.- García-Juárez G, Ramírez-Bribiesca J E, Hernández-Vázquez M, Hernández-Calva L M, Díaz-Aparicio E, Orozco-Bolaños H. Risk analysis of brucellosis in the state of Tlaxcala. Salud Publica Mex 2014; 56 (4): 355-62.

5.- Wyatt H. Lessons from the history of brucellosis. Rev Sci Tech 2013; 32 (1): 17-25.

6.- Vassallo D. The corps disease: brucellosis and its historical association with the Royal Army Medical Corps. J R Army Med Corps 1992; 138 (3): $140-50$.

7.- Moreno E. Retrospective and prospective perspectives on zoonotic brucellosis. Front Microbiol 2014; 5 (213). doi: 10.3389/ fmicb.2014.00213. e Collection 2014.

8.- Mancini F R, Bella A, Graziani C, Marianelli C, Mughini-Gras L, Pasquali P, et al. Trends of human brucellosis in Italy, 1998-2010. Epidemiol Infect 2014; 142 (6): 1188-95.

9.- Jia P, Joyner A. Human brucellosis occurrences in inner Mongolia, China: a spatio-temporal distribution and ecological niche modeling approach. BMC Infect Dis 2015; 15: 36. doi: 10.1186/s12879-015-0763-9.

10.- Godfroid J, DeBolle X, Roop R M, O'Callaghan D, Tsolis R M, Baldwin C, et al. The quest for a true one health perspective of brucellosis. Rev Sci Tech 2014; 33 (2): 521-38.

11.- Scholz H C, Hubalek Z, Sedlácek I, Vergnaud G, Tomaso H, Al Dahouk S, et al. Brucella microti sp. nov. isolated from the common vole Microtus arvalis. Int J Syst Evol Microbiol 2008; 58 (2): 375-82.

12.- Godfroid J, Cloeckaert A, Liautard J P, Kohler S, Fretin D, Walravens K, et al. From the discovery of the Malta Fever's agent to the discovery of a marine mammal reservoir, brucellosis has continuously been a re-emerging zoonosis. Vet Res 2005; 36 (3): 313-26.

13.- Foster G, Osterman, BS, Godfroid J, Jacques I, Cloeckaert A. Brucella ceti sp. nov.and Brucella pinnipedialis sp. nov. for Brucella strains with cetaceans and seals as their preferred hosts. Int J Syst Evol Microbiol 2007; 57 (11): 2688-93.
14.- Scholz H C, Nöckler K, Gollner C, Bahn P, Vergnaud G, Tomaso H, et al. Brucella inopinata sp. nov., isolated from a breast implant infection. Int J Syst Evol Microbiol 2010; 60 (4): 801-8.

15.- Whatmore A M, Davison N, Cloeckaert A, Al Dahouk S, Zygmunt M S, Brew S D, et al. Brucella papionis sp. nov. isolated from baboons (Papio spp.) Int J Syst Evol Microbiol 2014; 64 (12): 4120-8.

16.- Scholz H C, Revilla-Fernández S, Al Dahouk S, Hammerl J A, Zygmunt M S, Cloeckaert A, et al. Brucella vulpissp. nov. a novel Brucella species isolated from mandibular lymph nodes of red foxes (Vulpes vulpes) in Austria. Int J Syst Evol Microbiol 2016; Feb 29. Doi 10.10997IJSEM.0.000998. [Epubahead of print].

17.- Swai E S, Schoonman L. Human brucellosis: seroprevalence and risk factors related to high risk occupational groups in Tanga Municipality, Tanzania. Zoonoses Public Health 2009; 56: 183-7.

18.- Ponce de León-Rosales S, Lazcano-Ponce E, Rangel-Frausto M, Sosa Lazcano L A, Huerta-Jiménez M A. Bioterrorist: notes for an agenda in case of the unexpected. Salud Publica Mex 2001; 43: 589-603.

19.- Traxler R M, Lehman M W, Bosserman E A, Guerra M A, Smith T L. A literature review of laboratory-acquired brucellosis. J Clin Microbiol 2013; 51 (9): 3055-62.

20.- Baron E J, Miller J M. Bacterial and fungal infections among diagnostic laboratory workers: evaluating the risk. Diagn Microbiol Infect Dis 2008; 60: 241-6.

21.- Robichaud S, Libman M, Behr M, Rubin E. Prevention of laboratory-acquired brucellosis. Clin Infect Dis 2004; 38: e119-22.

22.- Bouza E, Sánchez-Carrilo C, Gómez S H, González J M. Spanish Co-operative Group for the Study of laboratory-acquired brucellosis. Laboratory-acquired brucellosis: a Spanish national survey. J Hosp Infect 2005; 61: 80-3.

23.- Atluri V L, Xavier M N, de Jong M F, den Hartigh A B, Tsolis R M. Interactions of the human pathogenic Brucella species with their hosts. Annu Rev Microbiol 2011; 65: 523-41.

24.- Martirosyan A, Moreno E, Gorvel J P. An evolutionary strategy for a stealthy intracellular Brucella pathogen. Immunol Rev 2011; 240 (1): 211-34.

25.- Dorneles E M, Teixeira-Carvalho A, Araújo M S, Sriranganathan N, Lage A P. Immune response triggered by Brucella abortus following infection or vaccination. Vaccine 2015; 17 (31): 3659-66.

26.- Skendros P, Boura P. Immunity to brucellosis. Rev Sci Tech 2013; 32 (1): 137-47.

27.- Rambow-Larsen A A, Petersen E M, Gourley C R, Splitter G A. Brucella regulators: self-control in a hostile environment. Trends Microbiol
2009; 17 (8): 371-7.

28.- Alsubaje S, Almuneef M, Alshaalan M, Balkhy H, Albanyan E, Atola S, et al. Acute brucellosis in Saudi families: relationship between brucella serology and clinical symptoms. Int $\mathrm{J}$ Infect Dis 2005; 9 (4): 218-24.

29.- Álvarez J, Sáez J L, García N, Serrat C, PérezSancho M, González S, et al. Management of an outbreak of brucellosis due to B. melitensis in dairy cattle in Spain. Res Vet Sci 2011; 90: 208-11.

30.- McDermott J, Grace D, Zinsstag J. Economics of brucellosis impact and control in low-income countries. Rev Sci Tech 2013; 32 (1): 249-61.

31.- Sanz C, Sáez J L, Álvarez J, Cortés M, Pereira $\mathrm{G}$, Reyes A, et al. Mass vaccination as a complementary tool in the control of a severe outbreak of bovine bruucellosis due to Brucella abortus in Extremadura, Spain. Prev Vet Med 2010; 97: 119-25.

32.- Halliday J E, Allan K J, Ekwem D, Cleaveland S, Kazwala R R, Crump J A. Endemic zoonoses in the tropics: a public health problem hiding in plain sight. Vet Rec 2015; 176: 220-5.

33.- Gul S T, Khan A, Ahmad M, Rizvi F, Shahzad A, Hussain I. Epidemiology of brucellosis at different livestock farms in the Punjab, Pakistan. PakVet J 2015; 35 (3): 309-14.

34.- Zabala C, Barragán V, Trueba G. Presencia de Brucella sp. en cabras de la ciudad de Quito, provincia de Pichincha, Ecuador. Avances 2012; 4 (2): B9-B11. DOI: 10.18272/aci.v4i2.100.

35.- Kumar A. Brucellosis: need of public health intervention in rural India. Prilozi 2010; 31 (1): 219-31.

36.- Agasthya A, Isloor S, Prabhudas K. Brucellosis in high risk group individuals. Indian J Med Microbiol 2007; 25: 28-31.

37.- Arnow P M, Smaron M, Ormiste V. Brucellosis in a group of travelers to Spain. JAMA 1984; 251 (4): 505-7.

38.- Tsou T P, Mu J J. Brucellosis: a neglected but existing threat to travelers and laboratory personnel in Taiwan. J Formos Med Assoc 2012; 11 (7): 353-4.

39.- Brough H A, Solomon A W, Wall R A, Isaza F, Pasvol G. Brucellosis acquired by eating imported cheese. J Pedriatr Child Health 2011; 47: 840-1.

40.- Ramos J M, Bernal E, Esguevillas T, López-García P, Gaztambide M S, Gutiérrez F. Non-imported brucellosis outbreak from unpasteurized raw milk in Moroccan immigrants in Spain. Epidemiol Infect 2008; 136 (11): 1552-5.

41.- Morales-García M R, García-Méndez N, Regalado-Jacobo S D, López-Merino A, Contreras-Rodríguez A. Clinical, serological and polymerase chain reaction follow-up of a family with brucellosis. Rev Chilena Infectol 2014; 31 (4): 425-33.

42.- Mantur B G, Mulimani M S, Bidari L H, 
Akki A S, Tikare N V. Bacteremia is as unpredcitable as clinical manifestations in humans brucellosis. Int J Infect Dis 2008; 12: 303-7.

43.- DOF. Diario Oficial de la Federación, 14 de marzo de 2012. NOM-022-SSA2-2012. Para la prevención y control de la brucelosis en el hombre, en el primer nivel de atención. Disponible: http://dof.gob.mx/nota_detalle.php? codigo $=5258723 \&$ fecha $=11 / 07 / 2012$ (Acceso el 14 abril de 2016).

44.- Sofian M, Aghakhani A, Velayati AA, Banifazl M, Eslamifar A, Ramezani A. Risk factors for human brucellosis in Iran: a case-control study. Int J Infect Dis 2008; 12 (2): 157-61.

45.- Tzaneva V, Ivanova S, Georgieva M, Tasheva E. Investigation of the spread of brucellosis among human and animal populations in southeastern Bulgaria, 2007. Euro Surveill 2009; 14 (17). pii: 19187.
46.- Flores-Figueroa J, Okhuysen P C, von Sonnenburg F, DuPont H L, Libman M D, Keystone J S, et al. Patterns of illness in travelers visiting Mexico and Central America: the Geo Sentinel experience. Clin Infect Dis 2011; 53 (6): 523-31.

47.- COFEPRIS. Comisión Federal para la protección contra Riesgos Sanitarios. Programa de Acción Específico 2007-2012. Secretaría de Salud. Disponible: http://www.cofepris.gob. $\mathrm{mx} /$ cofepris/Documents/QueEsCOFEPRIS/ prgaccion.pdf. Página 26. (Acceso el 14 de abril de 2016).

48.- Secretaria de Salud. Dirección General de Epidemiología. (SUIVE). Sistema Único de Información para la Vigilancia Epidemiológica Boletín epidemiológico. Cuadro 8 Brucelosis. Disponible: http://www.epidemiologia.salud. gob.mx/dgae/boletin/intd_boletin.html. (Acceso el 14 de abril de 2016).
49.- Morales-García M R, López-Méndez J, Pless R, García-Morales E, Kosanke H Hernández-Castro R, et al. Brucellosis outbreak in a rural endemic region of Mexico-a comprehensive investigation. Vet Ital 2015; 51 (3): $185-90$

50.- Oceguera M D, Bruce M, Frankena K, Udo H, van der Zijpp A, Rushton J. Financial analysis of brucellosis control for small-scale goat farming in the Bajío region, Mexico. Prev Vet Med 2015; 118 (4): 247-59.

51.- García-Juárez G, Ramírez-Bribiesca J E, Hernández-Vázquez M, Hernández-Calva L M, Orozco-Bolaños H, Jiménez L J. Brucellosis: socioeconomic status family and quality of life in two contrasting areas of the state of Tlaxcala, México. Estudios sociales (Hermosillo, Son.) 2013; 21 (41): 239-59. Disponible en: http:// www.scielo.org.mx/pdf/estsoc/v21n41/ v21n41a10.pdf. (Acceso el 14 de abril de 2016). 